\title{
Indicadores de sustentabilidade socioeconômica e ambiental dos assentamentos da reforma agrária em Mato Grosso - Brasil
}

\author{
Indicators of socio-economic and environmental sustainability of agrarian reform settlements in \\ Mato Grosso - Brazil \\ Indicadores de sostenibilidad socioeconómica y ambiental de los asentamientos de reforma agraria \\ en Mato Grosso - Brasil
}

\section{Resumo}

Os assentamentos rurais da reforma agrária se constrituem em unidades produtivas concebidas que visam a sustentabilidade mbiental e a melhoria da qualidade de vida no meior rural. Surge, então, a necessidade de avaliação da sustentabilidade com três focos principais, o ambiental, o econômico e social. Diante disso, o objetivo deste estudo foi analisar os indicadores de sustentabilidade socioeconômica e ambiental dos assentamentos rurais no Estado de Mato Grosso. Para tanto, foram realizados levantamentos bibliográficos e documentais acerca do desenvolvimento sustentável, reforma agrária e assentamentos rurais. Metodologicamente, é utilizado o Barômetro de Sustentabilidade, ferramenta que reúne indicadores de dimensões relacionadas ao bem-estar do meio ambiente e bem-estar humano. Foram analisados, 3.857 lotes rurais, de 39 assentamentos, totalizando 11 municípios do estado de Mato Grosso. Os resultados mostram que o grau de sustentabilidade obtido foi de grau Intermediário, em uma escala de Ruim (Insustentável), Pobre (Potencialmente Insustentável), Médio (Intermediário), Bom (Potencialmente sustentável) e Muito Bom (Sustentável). Os fatores que influenciam negativamente são o pouco acesso a programas sociogovernamentais e o alto índice de utilização de agrotóxicos nas áreas de produção dos assentados. Dentre os fatores que inlfuenciam positivamente tem-se o acesso à saúde e à educação.

Palavras-chave: Agricultura familiar, Assentamentos rurais, Barômetro da sustentabilidade.

\begin{abstract}
The rural settlements of the agrarian reform are constituted in production units conceived to aim at environmental sustainability and improving the quality of life in the rural environment. It arises, therefore, due to the need for an endorsement of sustainability with three main focuses, either environmental, or economic and social. The purpose of this study was to analyze the socioeconomic and environmental sustainability indicators of two rural assemblies in the State of Mato Grosso. To this end, bibliographic and documentary surveys have been carried out on sustainable development, agrarian reform and rural settlements. Methodologically, it is used the Sustainability Barometer, a
\end{abstract}


ferramenta that gathers indicators of dimensions related to being in the environment and being human. Foram analyzed, 3,857 rural lots, of 39 settlements, totaling 11 municipalities of the state of Mato Grosso. The results show that the degree of sustainability obtained was obtained from an Intermediate degree, on a scale: Bad (Unsustainable), Poor (Potentially Insustentável), Medium (Intermediate), Bom (Potentially sustainable) and Very Good (Sustainable). Factors that negatively influence only or little access to socio-governmental programs and a high rate of use of pesticides in two established production areas. Among the factors that positively influence your health and education.

Keywords: Family agriculture, Rural settlements, Sustainability Barometer.

\section{Resumen}

Los asentamientos rurales de la reforma agraria se constituyen en unidades productivas concebidas para apuntar a la sostenibilidad ambiental y mejorar la calidad de vida en el medio rural. Por tanto, es necesario evaluar la sostenibilidad con tres enfoques principales, el medioambiental, el económico y el social. Por tanto, el objetivo de este estudio fue analizar los indicadores de sostenibilidad socioeconómica y ambiental de los asentamientos rurales del Estado de Mato Grosso. Por ello, se realizaron levantamientos bibliográficos y documentales sobre desarrollo sustentable, reforma agraria y asentamientos rurales. Metodológicamente, se utiliza el Barómetro de Sostenibilidad, una herramienta que recoge indicadores de dimensiones relacionadas con el bienestar del medio ambiente y el bienestar humano. Se analizaron un total de 3.857 lotes rurales de 39 asentamientos, totalizando 11 municipios del estado de Mato Grosso. Los resultados muestran que el grado de sostenibilidad obtenido fue Intermedio, en una escala de Mala (Insostenible), Deficiente (Potencialmente insostenible), Medio (Intermedio), Bueno (Potencialmente sostenible) y Muy bueno (Sostenible). Los factores que influyen negativamente son el acceso limitado a programas socio-gubernamentales y la alta tasa de uso de plaguicidas en las áreas de producción de los pobladores. Entre los factores que influyen positivamente está el acceso a la salud y la educación.

Palabras clave: Agricultura familiar, Asentamientos Rurales, Barómetro de sostenibilidad.

\section{Introdução}

A reforma agrária apresentou maior visibilidade nas temáticas atuais, uma vez que se trata de uma oportunidade valiosa para os pequenos agricultores rurais do Brasil já que a elevada concentração de terra dificulta a justiça social, e uma grande parcela da população rural fica na fronteira da equidade do poder econômico e social.

A partir disso, unindo ao tema da reforma agrária e considerando que o desenvolvimento sustentável propõe o equilíbrio do uso dos recursos naturais, com objetivo de garantir que eles possam continuar existindo pelas próximas gerações, e que através dela é possível reduzir problemas como aquecimento global, queimadas e o fim dos recursos naturais, a sustentabilidade surge com três focos principais, o ambiental, o econômico e social, dessa forma o ambiente deve estar em perfeita sincronia para que seja sustentável.

Considerando esses questionamentos em relação a sustentabilidade associada aos pequenos produtores rurais, definiuse o seguinte problema de pesquisa: qual o impacto social, ambiental e econômico na criação de assentamentos rurais da reforma agrária? Após levantamento teórico de estudos anteriores baseados em Rossetto et al. (2020) e Silva e Vieira (2016), partiu-se do pressuposto de que os impactos sejam variáveis, dependem dos aspectos analisados, uma vez que pode haver pontos em excelente situação e pontos negativos.

Então, através desse viés, percebeu-se a necessidade de associar os conceitos e significados de sustentabilidade e reforma agrária, dessa forma o estudo teve por objetivo analisar os indicadores de sustentabilidade socioeconômica e ambiental dos assentamentos rurais no Estado de Mato Grosso, utilizando como fonte de informações o banco de dados do Projeto Diagnóstico para Regularização Ambiental dos Assentamentos de Reforma Agrária (RADIS/UFMT), produzido pela Universidade Federal de Mato Grosso (UFMT) e o Instituto Nacional de Colonização e Reforma Agrária (INCRA). Para tanto, foram estabelecidos os seguintes objetivos específicos: a) Levantar as unidades familiares e assentamentos para análise do trabalho; b) Analisar a sustentabilidade dos assentamentos da Reforma Agrária no âmbito do Projeto RADIS-UFMT; c) Realizar considerações para tomadores de decisões de políticas públicas relacionadas às questões fundiárias do Estado de Mato Grosso.

O Projeto RADIS/UFMT fornece um banco de dados para análise da condição social, econômica e ambiental dos 
assentamentos de reforma agrária no estado de Mato Grosso e tem como objetivos, a regularização ambiental dos assentamentos rurais por meio do Cadastro Ambiental Rural (CAR), o Diagnóstico de Sistemas Agrários e a elaboração de pesquisas acadêmicas

\section{Referencial Teórico}

\section{A importância da reforma agrária e do desenvolvimento sustentável}

Busca-se esclarecer os principais conceitos que norteiam a pesquisa, como reforma agrária, assentamentos rurais, desenvolvimento sustentável e barômetro da sustentabilidade, dessa forma, partindo da ideia de Sá-Silva, Almeida e Guindani (2009) foi realizada uma pesquisa bibliográfica e documental, pois a partir dela é possível ampliar a compreensão de um objeto que necessita de contextualização.

Segundo Carvalho et al. (2009), a reforma agrária constituiu-se como o principal mecanismo de enfrentamento da elevada concentração de terras, uma vez que na década de 1990, o neoliberalismo propunha a distribuição de terras via livre mercado, de forma que não beneficiou os pequenos agricultores. A transformação rural, surgiu a partir do momento em que as políticas com intenção de elevar a equidade e redistribuir o poder econômico foram alcançadas.

Nessa perspectiva, a Política Nacional de Reforma Agrária foi e tem sido um instrumento de gestão pública que realiza processos de reorganização fundiária, por meio da criação de comunidades de assentamentos rurais para à população que não possui acesso à posse da terra. A Reforma Agrária está prevista na Constituição Brasileira, que atribui ao Governo Federal a função de desapropriar terras que não estão cumprindo sua função social, de forma racional e adequada, preservando o meio ambiente e observando as legislações (Brasil, 1988).

Em concordância, e considerando a trajetória brasileira em relação a distribuição de terras, é possível perceber que as políticas empregadas causaram uma elevada concentração de terras, de forma que essa situação dificulta o desenvolvimento sustentável (Carvalho et al., 2009). Segundo Guerra (2002), os assentamentos rurais possuem uma organização familiar muito complexa, baseada em trabalho agrícola, e preocupação ecológico-ambiental, isso torna medir a sustentabilidade uma tarefa complexa, uma vez que Silva (2001) afirma que, inicialmente, a avaliação dos assentamentos era exclusivamente pelo desenvolvimento econômico. Diante disso, o desenvolvimento sustentável tem se tornado um tema de extrema relevância no contexto atual da sociedade, uma vez que relaciona a forma que o ser humano lida com o meio ambiente e as consequências que esse uso gera nas futuras gerações.

Através da implantação do desenvolvimento sustentável tanto nos países desenvolvidos, quanto em desenvolvimento, é possível atender as necessidades básicas e garantir a melhora da qualidade de vida atual, sem comprometer o futuro da população devido a padrões de vida com excessos (Brundtland, 1987). No entanto, a partir da divulgação do "Relatório Brundtland" realizado pela Comissão Mundial sobre Meio Ambiente e Desenvolvimento em 1987, o conceito do termo se tornou um marco nas discussões de temas sociais, políticos e econômicos, uma vez que contribui para o processo de mudança em relação ao abuso de recursos ambientais (Brundtland, 1987).

A sustentabilidade dos assentamentos rurais, tem sido alvo de questionamentos acerca dos potenciais impactos ambientais decorrentes da produção, bem como acerca da sustentabilidade socioeconômica dos assentados. Entretanto, é essencial ressaltar a importância dos assentamentos rurais para a distribuição de terras, já que através dessa distribuição é possível reduzir o desmatamento e implantar com maior facilidade a agricultura familiar.

\section{Barômetro da Sustentabilidade}

Inicialmente, não se tinha outro tipo de preocupação em relação aos assentamentos rurais, a não ser o desempenho econômico, considerando apenas a renda agrícola (Silva, 2001). Dessa forma, a partir da necessidade de ter uma visão 
multidimensional, para que seja possível analisar indicadores sociais, econômicos, ambientais e políticos, inicia-se as analises através do Índice Barômetro da Sustentabilidade.

O Barômetro da Sustentabilidade é uma ferramenta de avaliação que tem o objetivo de combinar, agrupar e analisar inúmeros indicadores relacionados a sustentabilidade, em diversas dimensões, com foco principal no meio-ambiente e o bemestar humano (Kronemberger, Carvalho \& Junior, 2008).

Trata-se de uma metodologia desenvolvida por Prescott-Allen (1997), com patrocínio do IUCN (Internacional Union for Conservation of Nature) e do IRDC (Internacional Research Development Center) e a ferramenta pode apresentar em sua estrutura quatro dimensões: Social, Econômica, Institucional e Ambiental, e por se tratar de um método flexível é possível adaptá-la a necessidade do usuário (Kronemberger et al, 2008).

Ainda, ao se tratar do Barômetro da Sustentabilidade, Rossetto et al. (2020) apresenta o desenvolvimento rural na perspectiva da sustentabilidade socioambiental, visando identificar os atributos do ambiente rural, da economia, e das características sociais dos assentamentos do estado de Minas Gerais, no qual, para os 28 assentamentos estudados, foi apurado grau 57 na dimensão do meio ambiente, considerado como intermediário, entretanto, quando avaliada a dimensão socioeconômica apresentou grau 30, definido como insustentável, dessa forma, o Barômetro da Sustentabilidade de Minas Geras apresentou como condição insustentável (Rossetto et al, 2020). Esses mesmo autores consideram que, diante desse cenário, há medidas que precisam ser implantadas para melhorar o desenvolvimento sustentável dos assentamentos, tais como melhorias sanitárias, incentivo de práticas conservacionistas, através de treinamento dos assentados, de maneira que aprendam noções de preservação do meio ambiente, e principalmente o fortalecimento das cooperativas e associações para promover o aumento da renda e da escolaridade da população local.

\section{Metodologia}

A metodologia adotada para o desenvolvimento do presente trabalho apoiou-se em Rossetto et al (2020) e considerada por Prescott-Allen $(1997 ; 2001)$ como uma ferramenta para medir e comunicar o bem-estar de uma sociedade e progredir em direção a sustentabilidade.

O universo da pesquisa consiste dos assentamentos pesquisados pelo Projeto RADIS/UFMT, em Mato Grosso, entre 2019 e 2020, totalizando 11 municípios, 39 assentamentos e 3.857 lotes rurais, localizados nas mesorregiões geográficas de Centro-Sul Mato-Grossense e Sudoeste Mato-Grossense (Tabela 1). 
Tabela 1 - Assentamentos e municípios que compõem a amostra da pesquisa.

\begin{tabular}{|c|c|c|c|}
\hline Mesorregiões & Municípios & Projeto de Assentamento (PA) & Número de Lotes \\
\hline \multirow[t]{20}{*}{ Sudoeste Mato-Grossense } & Barra do Bugres & PA Cabaças & 147 \\
\hline & Jauru & PA Corgão & 93 \\
\hline & Jauru & PA Mirassolzinho & 299 \\
\hline & \multirow{3}{*}{ Mirassol D'Oeste } & PA Santa Helena II & 46 \\
\hline & & PA Silvio Rodrigues & 134 \\
\hline & & PA Roseli Nunes & 247 \\
\hline & \multirow{5}{*}{ Nova Olímpia } & PA Rio Branco & 69 \\
\hline & & PA Riozinho & 50 \\
\hline & & PA Usiel Pereira & 24 \\
\hline & & PA Vale do Sol & 33 \\
\hline & & PDS Nova Conquista & 39 \\
\hline & Nova Brasilandia & PA Santa Rosa I & 63 \\
\hline & & PA Rio Alegre & 175 \\
\hline & & PA Triunfo & 199 \\
\hline & São José dos Quatro Marcos & PA Florestan Fernandes & 141 \\
\hline & \multirow{2}{*}{ Tangará da Serra } & PA Antônio Conselheiro & 544 \\
\hline & & PA Triangulo & 74 \\
\hline & \multirow{3}{*}{$\begin{array}{l}\text { Vila Bela da Santíssima } \\
\text { Trindade }\end{array}$} & PA São Sebastião & 11 \\
\hline & & PA Seringal & 175 \\
\hline & & PA Ritinha & 99 \\
\hline \multirow[t]{19}{*}{ Centro-Sul Mato-Grossense } & \multirow{18}{*}{ Cáceres } & PA Barranqueira & 52 \\
\hline & & PA Bom sucesso & 9 \\
\hline & & PA Corixo & 51 \\
\hline & & PA Facão/Bom Jardim & 87 \\
\hline & & PA Ipê Roxo & 8 \\
\hline & & PA Jatobá & 20 \\
\hline & & PA Katira & 19 \\
\hline & & PA Laranjeira I & 71 \\
\hline & & PA Laranjeira II & 22 \\
\hline & & PA Limoeiro & 85 \\
\hline & & PA Margarida Alves & 123 \\
\hline & & PA Mirassolzinho II & 50 \\
\hline & & PA Nova Esperança & 36 \\
\hline & & PA Paiol & 177 \\
\hline & & PA Rancho da Saudade & 41 \\
\hline & & PA Recompensa II & 19 \\
\hline & & PA Sadia Vale Verde & 281 \\
\hline & & PA Sapicuá & 36 \\
\hline & Porto Esperidião & PA Papiro & 8 \\
\hline Total & 11 & 39 & 3857 \\
\hline
\end{tabular}

Fonte: Autores. 
ambiental, econômica e social da sustentabilidade, considerando-se como sistema os 39 assentamentos rurais pesquisados, e como subsistemas, os 3.857 lotes rurais. Assim, é utilizado um conjunto de sete temas - saneamento básico, práticas de manejo do ambiente natural, usos de terra, renda, bem-estar social, saúde e educação - (Figura 1) e dezessete indicadores, para que as fontes/valores e objetivos de referências para a construção da Escala de Desempenho Local, contemplando parâmetros nacionais, estaduais e globais de referências e obedecendo o critério da flexibilidade e relevância para a realidade a ser analisados.

Figura 1 - Estrutura dos indicadores de sustentabilidade dos assentamentos rurais do Estado de Mato Grosso.

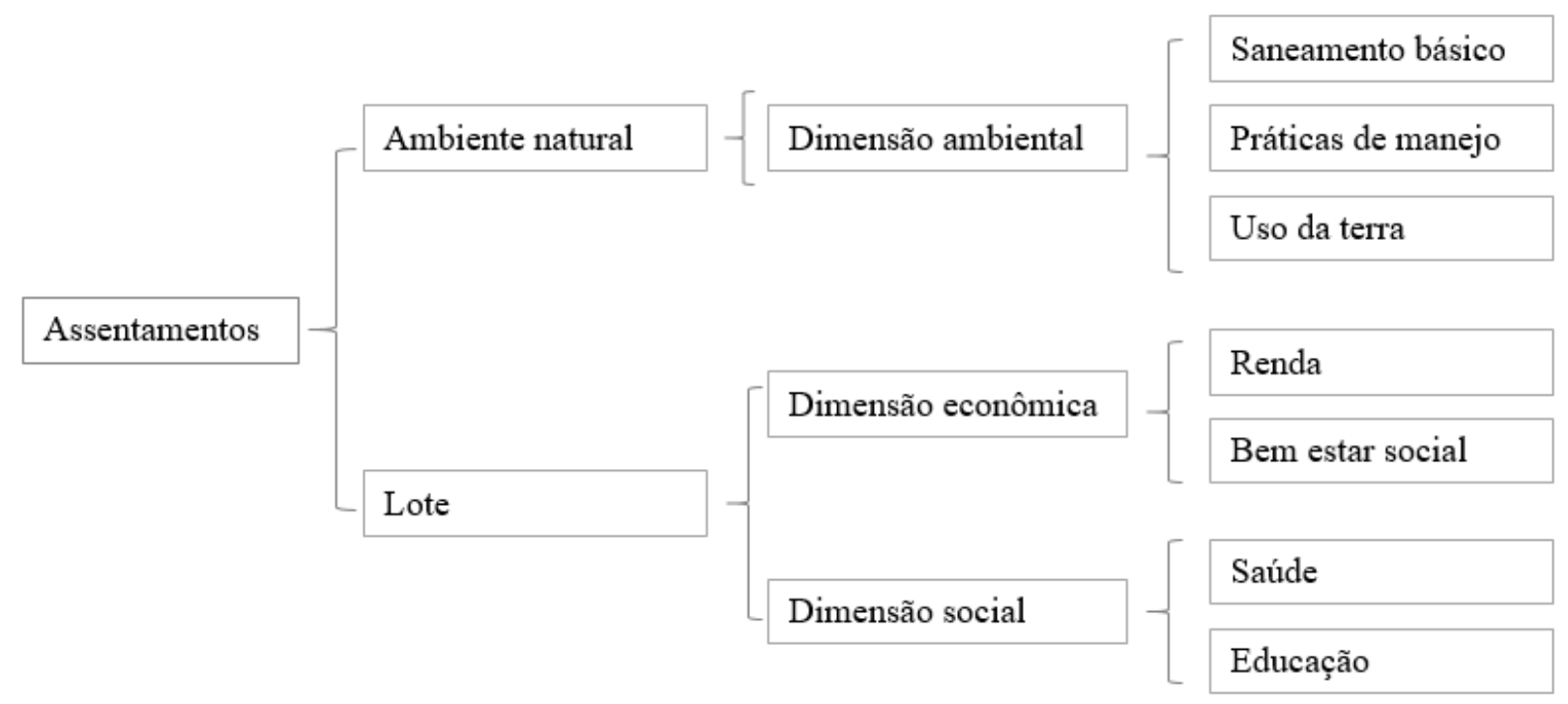

Fonte: Rossetto et al (2020).

Os indicadores adotados nesse estudo baseiam-se em Rossetto et al. (2020), em que é realizada uma análise nos âmbitos social, econômico e ambiental, pois para Gallopin (1996) os indicadores precisam ser compreensíveis e transparentes. Os autores Gallopin (1996) e Van Bellen (2007) consideram que os indicadores de desenvolvimento sustentável devem seguir alguns requisitos: os valores dos indicadores devem ser mensuráveis (ou observáveis); disponibilidade de dados; a metodologia para coleta e o processamento de dados precisam ser limpos, transparentes e padronizados.

A análise dos Indicadores de Sustentabilidade foi realizada de forma a considerar os dados mais relevantes, seja positiva ou negativamente em cada dimensão e de acordo com as referências estabelecidas para a escala de desempenho (Quadro 1).

Em seguida, na Tabela 2, foi atribuída uma Escala de Desempenho Local (EDL) para cada indicador, através da média dos valores atribuídos individualmente a cada um deles. A escala de sustentabilidade pode ser crescente ou decrescente de acordo com a relação estabelecida entre o indicador e a sustentabilidade por ela expressa (Rossetto et al., 2020). 
Quadro 1 - Indicadores de desenvolvimento sustentável e referências para a construção da Escala de Desempenho.

\begin{tabular}{|c|c|}
\hline Indicadores & Referências \\
\hline Número de moradias com fossa/sumidouro (\%) & \multirow{3}{*}{$\begin{array}{l}\text { - Lei Federal } \mathrm{n}^{\circ} 11.445 \text {, de } 5 \text { de janeiro de } 2007 \text { : Estabelece } \\
\text { asdiretrizes nacionais para o saneamento básico princípio } \\
\text { fundamental de universalização do acesso ( } 100 \% \text { da população } \\
\text { atendida) (Brasil, 2007): }\end{array}$} \\
\hline $\begin{array}{l}\text { Número de Moradias que não possuem sistema de esgoto } \\
\text { sanitário }(\%)\end{array}$ & \\
\hline $\begin{array}{l}\text { Número de Moradias que possuem fossa séptica ou } \\
\text { realizam tratamentoecológico }(\%)\end{array}$ & \\
\hline Número de moradias que tem acesso à água ( \%) & \multirow{3}{*}{$\begin{array}{l}\text { - Lei Estadual no } 11.088 \text {, de } 09 \text { de março de 2020: Dispõe } \\
\text { sobre a Política Estadual de Recursos Hídricos, institui o } \\
\text { Sistema Estadual de Recursos Hídricos e dá outras } \\
\text { providências (Mato Grosso, 2020) } \\
\text { - Resolução no 64/292 (2010) (ONU, 2010) }\end{array}$} \\
\hline $\begin{array}{l}\text { Número de moradias que retiram águadiretamente de } \\
\text { nascentes ou rios }(\%)\end{array}$ & \\
\hline $\begin{array}{l}\text { Número de moradias que retiram água de poço coletivo; } \\
\text { poço semiartesiano; poço artesiano }(\%)\end{array}$ & \\
\hline $\begin{array}{l}\text { Número de lotes que desenvolvem práticas de } \\
\text { conservação ambiental }(\%)\end{array}$ & $\begin{array}{l}\text { - Lei Complementar n }{ }^{\circ} 38 \text {, de } 21 \text { de novembro de 1995: Dispõe } \\
\text { sobre o Código Estadual do Meio Ambiente e dá outras } \\
\text { providências (Mato Grosso, 1995) }\end{array}$ \\
\hline $\begin{array}{l}\text { Número de lotes que desenvolvem práticas nocivas ao } \\
\text { meio ambiente }(\%)\end{array}$ & $\begin{array}{l}\text { - Conjuntura de Recursos Hídricos - 2019: MatoGrosso } \\
\text { comercializou 100.638ton de agrotóxicos 2017 (ANA, 2019) }\end{array}$ \\
\hline Total de Área Degradads (hectars) & $\begin{array}{l}\text {-Área total dos lotes de assentamentos levantados pelo Projeto } \\
\text { RADIS/UFMT } \\
\text {-Área total do estado de Mato Grosso: } 903.207,050 \mathrm{Km}^{2} \\
\text { (Brasil, 2021) } \\
\text {-Área rural total do Estado de Mato Grosso: } 37.900 .000,00 \mathrm{ha} \\
\text { (IBGE, 2017) }\end{array}$ \\
\hline Total de Área de Mata Nativa (hectars) & $\begin{array}{l}\text {-Área total de mata nativa ou floresta natural na área rural do } \\
\text { Estado de Mato Grosso é de 17.481.696 hectares em } 118.679 \\
\text { estabelecimentos (IBGE, 2017) }\end{array}$ \\
\hline Total de Área Florestada (hectares) & $\begin{array}{l}\text {-Área total de área florestada na área rural do Estado de Mato } \\
\text { Grosso é de 201.102ha (IBGE, 2017) }\end{array}$ \\
\hline $\begin{array}{l}\text { Número de agricultores que recebem renda bruta anual } \\
\text { total até } \mathrm{R} \$ 20.000,00(\%)\end{array}$ & \multirow{6}{*}{$\begin{array}{l}\text {-Atlas de Desenvolvimento Humano do Brasil: a renda média é } \\
\text { de R } \$ 808,99 \text { mensais em Mato Grosso (PNUD, 2010) } \\
\text { - Percentual de pessoas com } 18 \text { anos ou mais com } \\
\text { fundamentalcompleto no Estado de Mato Grosso (IBGE, 2017) }\end{array}$} \\
\hline $\begin{array}{l}\text { Número de agricultores que adquirem renda com } \\
\text { atividades econômicas externas ao lote }(\%)\end{array}$ & \\
\hline $\begin{array}{l}\text { Número de famílias que recebem algum tipo de programa } \\
\text { social governamental }(\%)\end{array}$ & \\
\hline $\begin{array}{l}\text { Número de famílias que participam de } \\
\text { ssociação/cooperativas (\%) }\end{array}$ & \\
\hline $\begin{array}{l}\text { Número de entrevistados que possuem somente o nível } \\
\text { fundamental completo }(\%)\end{array}$ & \\
\hline Número de famílias que tem acesso a posto de saúde $(\%)$ & \\
\hline
\end{tabular}

Fonte: Autores. 
Tabela 2 - Escala de Desenvolvimento Local (EDL) de cada indicador.

\begin{tabular}{|c|c|c|c|c|c|}
\hline Indicadores (DLx) & $\begin{array}{c}\text { Ruim } \\
\text { Insustentável }\end{array}$ & \begin{tabular}{|c|} 
Pobre \\
Potencialmente \\
insustentável
\end{tabular} & $\begin{array}{c}\text { Médio } \\
\text { Intermediário }\end{array}$ & $\begin{array}{l}\text { Bom } \\
\text { Potencial- } \\
\text { mente } \\
\text { sustentável }\end{array}$ & $\begin{array}{l}\text { Muito Bom } \\
\text { Sustentável }\end{array}$ \\
\hline $\begin{array}{l}\text { Número de moradias com fossa/ } \\
\text { sumidouro }(\%)\end{array}$ & $0<\mathrm{DLx} \leq 10$ & $10<\mathrm{DLx} \leq 35$ & $35<\mathrm{DLx} \leq 65$ & $65<\mathrm{DLx} \leq 85$ & $85<$ DLx $\leq 100$ \\
\hline $\begin{array}{l}\text { Número de moradias que não possuem } \\
\text { sistema de esgoto sanitário }(\%)\end{array}$ & $100<\mathrm{DLx} \leq 85$ & $85<\mathrm{DLx} \leq 65$ & $65<\mathrm{DLx} \leq 35$ & $35<\mathrm{DLx} \leq 10$ & $10<\mathrm{DLx} \leq 0$ \\
\hline $\begin{array}{l}\text { Número de moradias que possuem fossa } \\
\text { séptica ou realizam tratamento ecológico } \\
(\%)\end{array}$ & $0<\mathrm{DLx} \leq 10$ & $10<\mathrm{DLx} \leq 35$ & $35<\mathrm{DLx} \leq 65$ & $65<\mathrm{DLx} \leq 85$ & $85<\mathrm{DLx} \leq 100$ \\
\hline $\begin{array}{l}\text { Número de moradias que tem acesso à } \\
\text { água (expresso em \%) }\end{array}$ & $0<\mathrm{DLx} \leq 5$ & $5<\mathrm{DLx} \leq 10$ & $10<\mathrm{DLx} \leq 40$ & $40<\mathrm{DLx} \leq 80$ & $80<\mathrm{DLx} \leq 100$ \\
\hline $\begin{array}{l}\text { Número de moradias que retiram água } \\
\text { diretamente de nascentes ou rios }(\%)\end{array}$ & $100<\mathrm{DLx} \leq 90$ & $90<\mathrm{DLx} \leq 60$ & $60<\mathrm{DLx} \leq 10$ & $10<\mathrm{DLx} \leq 5$ & $5<\mathrm{DLx} \leq 0$ \\
\hline $\begin{array}{l}\text { Número de moradias que retiram água de } \\
\text { poço coletivo; poço semiartesiano; poço } \\
\text { artesiano }(\%)\end{array}$ & $0<\mathrm{DLx} \leq 5$ & $5<\mathrm{DLx} \leq 10$ & $10<\mathrm{DLx} \leq 40$ & $40<\mathrm{DLx} \leq 80$ & $80<\mathrm{DLx} \leq 100$ \\
\hline $\begin{array}{l}\text { Números de lotes que desenvolvem } \\
\text { práticas de conservação ambiental }(\%)\end{array}$ & $0<\mathrm{DLx} \leq 20$ & $20<\mathrm{DLx} \leq 40$ & $40<\mathrm{DLx} \leq 60$ & $60<\mathrm{DLx} \leq 80$ & $80<\mathrm{DLx} \leq 100$ \\
\hline $\begin{array}{l}\text { Números de lotes que desenvolvem } \\
\text { práticas nocivas ao meio ambiente }(\%)\end{array}$ & $100<\mathrm{DLx} \leq 60$ & $60<\mathrm{DLx} \leq 30$ & $30<\mathrm{DLx} \leq 20$ & $20<\mathrm{DLx} \leq 10$ & $10<\mathrm{DLx} \leq 0$ \\
\hline Total de Área Degradada (ha) & $100<\mathrm{DLx} \leq 50$ & $50<\mathrm{DLx} \leq 25$ & $25<\mathrm{DLx} \leq 20$ & $20<\mathrm{DLx} \leq 15$ & $15<\mathrm{DLx} \leq 0$ \\
\hline Total de Área de Mata Nativa (ha) & $0<\mathrm{DLx} \leq 15$ & $15<\mathrm{DLx} \leq 25$ & $25<\mathrm{DLx} \leq 40$ & $40<\mathrm{DLx} \leq 65$ & $65<\mathrm{DLx} \leq 100$ \\
\hline Total de Área Florestada (ha) & $0<\mathrm{DLx} \leq 15$ & $15<\mathrm{DLx} \leq 25$ & $25<\mathrm{DLx} \leq 40$ & $40<\mathrm{DLx} \leq 65$ & $65<\mathrm{DLx} \leq 100$ \\
\hline $\begin{array}{l}\text { Número de agricultores que recebem } \\
\text { renda bruta anual total até } \mathrm{R} \$ 20.000,00 \\
(\%)\end{array}$ & $100<\mathrm{DLx} \leq 80$ & $80<\mathrm{DLx} \leq 60$ & $60<\mathrm{DLx} \leq 40$ & $40<\mathrm{DLx} \leq 20$ & $20<$ DLx $\leq 0$ \\
\hline $\begin{array}{l}\text { Número de agricultores que adquirem } \\
\text { renda com atividades econômicas externas } \\
\text { ao lote }(\%)\end{array}$ & $0<\mathrm{DLx} \leq 50$ & $50<$ DL $x \leq 75$ & $75<\mathrm{DLx} \leq 80$ & $80<\mathrm{DLx} \leq 90$ & $90<\mathrm{DLx} \leq 100$ \\
\hline $\begin{array}{l}\text { Número de famílias que recebem algum } \\
\text { tipo de programa social } 1(\%)\end{array}$ & $0<\mathrm{DLx} \leq 25$ & $25<$ DLx $\leq 50$ & $50<\mathrm{DLx} \leq 75$ & $75<$ DLx $\leq 90$ & $90<\mathrm{DLx} \leq 100$ \\
\hline $\begin{array}{l}\text { Número de famílias que participam de } \\
\text { associação/cooperativas }(\%)\end{array}$ & $0<\mathrm{DLx} \leq 20$ & $20<$ DLx $\leq 40$ & $40<\mathrm{DLx} \leq 60$ & $60<\mathrm{DLx} \leq 80$ & $80<\mathrm{DLx} \leq 100$ \\
\hline $\begin{array}{l}\text { Número de entrevistados que possuem } \\
\text { somente o nível fundamental incompleto } \\
(\%)\end{array}$ & $0<\mathrm{DLx} \leq 20$ & $20<\mathrm{DLx} \leq 40$ & $40<\mathrm{DLx} \leq 60$ & $60<\mathrm{DLx} \leq 80$ & $80<\mathrm{DLx} \leq 100$ \\
\hline $\begin{array}{l}\text { Número de famílias que tem acesso a } \\
\text { posto de saúde }(\%)\end{array}$ & $0<\mathrm{DLx} \leq 20$ & $20<\mathrm{DLx} \leq 30$ & $30<\mathrm{DLx} \leq 50$ & $60<\mathrm{DLx} \leq 80$ & $80<\mathrm{DLx} \leq 100$ \\
\hline
\end{tabular}

Fonte: Rossetto et al (2020).

Os limites da Escala do Barômetro de Sustentabilidade (EBS) foram estabelecidos conforme a Figura 2. Após essa etapa, é possível observar na Figura 3 que o valor dos dados do indicador foi localizado no EDL, bem como os pontos anterior (DLA) e posterior (DLP). Os cinco valores numéricos das duas escalas (EDL e EBS) foram alinhados, determinando a posição relativa da EDL na escala EBS (Rossetto et al., 2020). 
Figura 2 - Transposição da EDL para a EBS de um indicador de relação a) crescente e b) decrescente com a sustentabilidade.

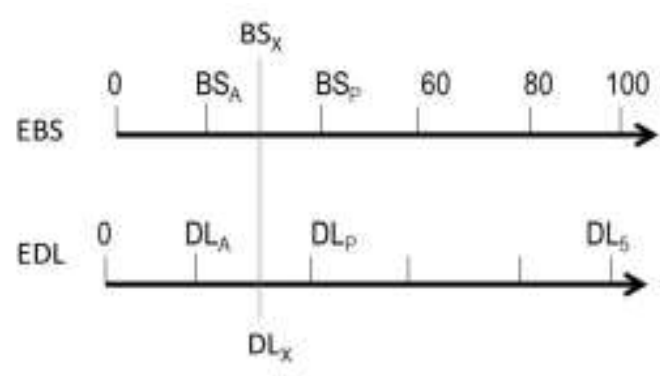

a)

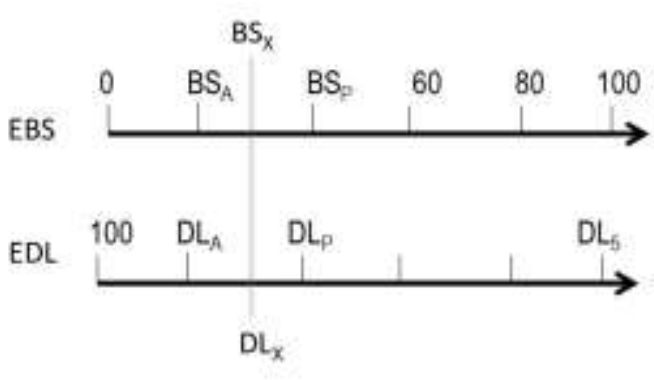

b)

Fonte: Adaptado de Prescott-Allen (1997; 2001).

Figura 3 - Escala do Barômetro da Sustentabilidade (EBS), onde BSx é o indicador local determinado.

$\mathbf{0}<\mathbf{B S x} \leq \mathbf{2 0}$

Ruim

(Insustentável)
$20<\mathrm{BSx} \leq 40$

Pobre
$40<\mathrm{BSx} \leq 60$

Médio
$60<\mathrm{BSx} \leq \mathbf{8 0}$

Bom

$\mathbf{8 0}<\mathrm{BSx} \leq \mathbf{1 0 0}$

Muito Bom

(Sustentável)

Fonte: Adaptado de Prescott-Allen (1997; 2001).

A transposição foi realizada por meio do processo matemático de interpolação linear simples, na qual um novo conjunto de dados $\left(\mathrm{BS}_{\mathrm{X}}\right)$ é construído a partir de dados conhecidos $\left(\mathrm{DL}_{\mathrm{A}}, \mathrm{DL}_{\mathrm{P}}, \mathrm{BS}_{\mathrm{A}}\right.$ e $\mathrm{BS}_{\mathrm{P}}$ e $\left.\mathrm{DL}_{\mathrm{X}}\right)$, conforme demonstrado pelas Equações 1 a 5, em que DLx é o valor do indicador de acordo com os dados do estudo; DL é o valor da escala imediatamente anterior a $\mathrm{DL}_{\mathrm{A}}$; DLP é o valor da escala imediatamente após $\mathrm{DL}_{\mathrm{A}} ; \mathrm{BS}_{\mathrm{X}}$ é o valor correspondente à transposição de $\mathrm{DL}_{\mathrm{A}}$ para $\mathrm{EB}_{\mathrm{S}} ; \mathrm{BS}_{\mathrm{A}}$ é o valor da escala imediatamente anterior a $\mathrm{BS}_{\mathrm{X}}$ e $\mathrm{BS}_{\mathrm{P}}$ é o valor da escala imediatamente após $\mathrm{BS}_{\mathrm{X}}$.

Equação 1

$\frac{D L_{A}-D L_{X}}{B S_{A}-B S_{X}}=\frac{D L_{A}-D L_{p}}{B S_{A}-B S_{P}}$

$\left(D L_{A}-D L_{X}\right) \cdot\left(B S_{A}-B S_{P}\right)=\left(D L_{A}-D L_{p}\right) \cdot\left(B S_{A}-B S_{X}\right)$

$\frac{\left(D L_{A}-D L_{X}\right) \cdot\left(B S_{A}-B S_{P}\right)}{\left(D L_{A}-D L_{p}\right)}=\left(B S_{A}-B S_{X}\right)$

$-B S_{X}=\frac{\left(D L_{A 1}-D L_{X}\right) \cdot\left(B S_{A}-B S_{P}\right)}{\left(D L_{A}-D L_{P}\right)}-B S_{A}$, multiplica-se por $(-1)$

$B S_{X}=\left[\frac{\left(D L_{A 1}-D L_{X}\right) \cdot\left(B S_{A}-B S_{P}\right)}{\left(D L_{A}-D L_{F}\right)} \cdot(-1)\right]+B S_{A}$

Considerando o Barômetro de Sustentabilidade (BS), as dimensões foram divididas em dois componentes: o índice de bem-estar social que considera as dimensões sociais e o econômicas e o índice de bem-estar ambiental, que considera gestão de recursos naturais (Prescott-Allen, 1997; 2001). Esses índices foram calculados através da média aritmética dos indicadores que os compõem. Em seguida, um gráfico bidimensional foi gerado que apresenta a situação de sustentabilidade local. A partir dessas referências é possível fazer uma análise dos indicadores e avaliar o quão sustentável se encontram os assentamentos rurais pesquisados no estado de Mato Grosso.
Equação 2

Equação 3

Equação 4

Equação 5 


\section{Resultados e Discussão}

O desenvolvimento sustentável pede uma análise múltipla, identificando pontos positivos e negativos dos indicadores, de forma que seja possível identificar o desenvolvimento do bem-estar ambiental e do bem-estar socioeconômico.

A Tabela 3 apresenta as notas obtidas na EDL e na EBS nas mesorregiões Centro-Sul Mato-grossense e Sudoeste Mato-grossense. Verificou-se que no indicador "número de residências com fossa/sumidouro", em nenhum assentamento pesquisado, os lotes possuíam fossa séptica e apresentaram nas mesorregiões Centro-Sul Mato-grossense e Sudoeste Matogrossense, as dimensões 80 e 79, respectivamente, considerado como grau Bom (Potencialmente sustentável). Esse critério admite que, a princípio, destinar os dejetos humanos para uma fossa simples (ou negra) ainda é melhor que tê-los correndo a céu aberto. Esses dois tipos de prática de fossa/sumidouro e esgoto a céu aberto são as predominantes nos assentamentos brasileiros, segundo Peroni, Carvalho e Lannes (2021).

O acesso declarado à água foi elevado, resultando em grau Muito Bom (Sustentável), nas duas mesorregiões, sendo maior o número de lotes que a retiram de poço que diretamente de rios ou nascentes. O grau desse sub-sistema foi de 61 e 47 , respectivamente, nas mesorregiões Centro-Sul Mato-grossense e Sudoeste Mato-grossense, grau Bom (Potencialmente sustentável).

Essa informação reflete a situação do Brasil, que tem dificuldades de cumprir o que está determinado em lei, uma vez que a Lei do Saneamento Básico (Brasil, 2017), referenciada no Quadro 1, determina que os serviços públicos de saneamento básico devem seguir alguns fundamentos, entre eles: universalização do acesso, integralidade, esgotamento sanitário. Diante disso, percebe-se que há um distanciamento da aplicação do esgotamento sanitário nessas regiões, apesar de que as localidades de pequeno porte estão integradas à referida lei, que determina o esgotamento sanitário como as atividades de infraestruturas e instalações operacionais de coleta, transporte, tratamento e disposição final adequados dos esgotos sanitários, desde as ligações prediais até o seu lançamento final no meio ambiente.

Em relação ao "número de moradias que não possuem sistema de esgoto sanitário" apresentaram os valores, 78 e 76, respectivamente, considerado Potencialmente sustentável em ambas as mesorregiões. E por último, o indicador "número de moradias que possuem fossa séptica ou realizam tratamento ecológico" obtiveram valor 0 nas duas mesorregiões, caracterizando como Insustentável.

Ainda considerando o tema Saneamento Básico, o indicador "número de moradias que tem acesso à água", apresentou nas mesorregiões Centro-Sul Mato-grossense e Sudoeste Mato-grossense os valores de 90 e 91, nessa ordem, considerados na escala como Sustentável, já o indicador número de moradias que retiram água diretamente de nascentes ou rios, os valores obtidos foram 48 e 37, considerado Intermediário e Potencialmente Insustentável. Para finalizar o tema saneamento básico, o último indicador "número de moradias que retiram água de poço coletivo; poço semiartesiano; poço artesiano" apresentou indicadores de valor 70 e 57, para centro-sul mato-grossense e sudoeste mato-grossense, nessa ordem.

Dessa forma, é possível perceber que, sem levar em consideração a forma que a água é obtida, o número de moradias que possuem acesso a água é excelente, pois conforme a Resolução nº 64/292 (ONU, 2010), citada no Quadro 1, o acesso a água e ao saneamento básico é um direito humano, e precisa ser disponibilizada potável e limpa. Entretanto, considerando o resultado dos indicadores de retirada de água diretamente das nascentes e rios, trate-se de um dado negativo, pois pode acontecer contaminações nas nascentes, já a retirada de água de poços artesianos precisa ser controlada, visto que se trata da água de lençóis freáticos e com a extração além da capacidade de reposição, o armazenamento dessa água pode se esgotar. A Lei Complementar no 38 (Brasil, 1995), também referenciada no Quadro 1 dispõe das penalidades caso haja infrações aos recursos hídricos, o ideal seria uma intervenção do governo, através de medidas que de fato sejam implantadas nas regiões dos assentamentos, gerando uma melhor qualidade de vida da população local, além de garantir que os recursos hídricos estão sendo utilizados de forma sustentável. 
Tabela 3 - Dimensão e Grau do Barômetro da Sustentabilidade da Mesorregião Centro-Sul Mato-grossense.

\begin{tabular}{|c|c|c|c|c|c|c|c|c|c|}
\hline \multirow{3}{*}{ 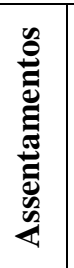 } & \multirow{3}{*}{ 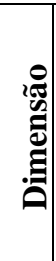 } & \multirow{3}{*}{ 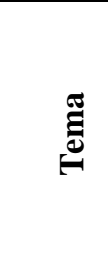 } & \multirow[b]{3}{*}{ Indicadores } & \multicolumn{6}{|c|}{ Graus na Mesorregião } \\
\hline & & & & \multicolumn{3}{|c|}{ Centro-Sul Mato-grossense } & \multicolumn{3}{|c|}{ Sudoeste Mato-Grossense } \\
\hline & & & & Dimensão & $\begin{array}{c}\text { Sub- } \\
\text { sistema }\end{array}$ & Sistema & Dimensão & $\begin{array}{l}\text { Sub- } \\
\text { sistema }\end{array}$ & Sistema \\
\hline \multirow{11}{*}{ 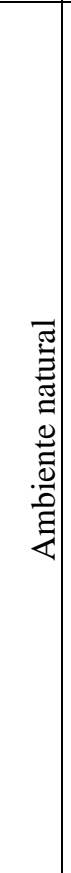 } & \multirow{11}{*}{ 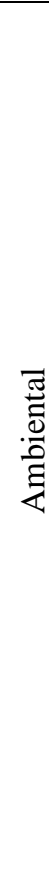 } & \multirow{6}{*}{ 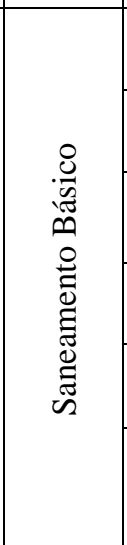 } & $\begin{array}{l}\text { Número de moradias combssa/ } \\
\text { sumidouro }\end{array}$ & 80 & \multirow{6}{*}{61} & \multirow{11}{*}{50} & 79 & \multirow[t]{6}{*}{57} & \multirow[t]{11}{*}{44} \\
\hline & & & $\begin{array}{l}\text { Número de moradias que não possuem } \\
\text { sistema de esgoto sanitário }\end{array}$ & 78 & & & 76 & & \\
\hline & & & $\begin{array}{l}\text { Número de moradias que possuem fossa } \\
\text { séptica ou realizam tratamento ecológico }\end{array}$ & 0 & & & 0 & & \\
\hline & & & $\begin{array}{l}\text { Número de moradias que tem acesso à } \\
\text { água }\end{array}$ & 90 & & & 91 & & \\
\hline & & & $\begin{array}{l}\text { Número de moradias que retiram água } \\
\text { diretamente de nascentes ou rios }\end{array}$ & 48 & & & 37 & & \\
\hline & & & $\begin{array}{l}\text { Número de moradias que retiramágua de } \\
\text { poço coletivo, poço semiartesiano ou } \\
\text { poço artesiano }\end{array}$ & 70 & & & 57 & & \\
\hline & & \multirow{2}{*}{ 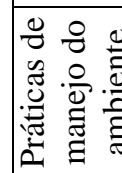 } & $\begin{array}{l}\text { Números de lotes que desenvolvem } \\
\text { práticas de conservação ambiental }\end{array}$ & 64 & \multirow[t]{2}{*}{37} & & 60 & \multirow[t]{2}{*}{35} & \\
\hline & & & $\begin{array}{l}\text { Números de lotes que desenvolvem } \\
\text { práticas nocivas ao meio ambiente }\end{array}$ & 11 & & & 9 & & \\
\hline & & \multirow{3}{*}{ 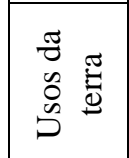 } & Total de áreas degradadas & 100 & \multirow{3}{*}{50} & & 100 & \multirow[t]{3}{*}{40} & \\
\hline & & & Total de área de mata nativa & 35 & & & 12 & & \\
\hline & & & Total de área florestada & 16 & & & 7 & & \\
\hline \multirow{6}{*}{ 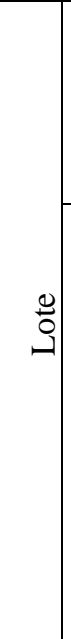 } & \multirow{2}{*}{ 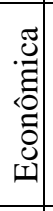 } & \multirow{2}{*}{$\frac{\pi}{\tilde{0}}$} & $\begin{array}{l}\text { Número de agricultores que recebem } \\
\text { renda bruta anual total até } \mathrm{R} \$ 20.000,00\end{array}$ & 56 & \multirow[b]{2}{*}{41} & \multirow{6}{*}{47} & 83 & 57 & 54 \\
\hline & & & $\begin{array}{l}\text { Número de agricultores que adquirem } \\
\text { renda com atividades econômicas } \\
\text { externas ao lote }\end{array}$ & 27 & & & 32 & & \\
\hline & \multirow{4}{*}{$\begin{array}{l}\cdot \vec{T} \\
0 \\
0 \\
n\end{array}$} & \multirow{2}{*}{ 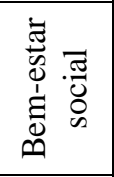 } & $\begin{array}{l}\text { Número de famílias que recebem algum } \\
\text { tipo de programa social governamental }\end{array}$ & 9 & \multirow{4}{*}{53} & & 6 & \multirow[t]{4}{*}{50} & \\
\hline & & & $\begin{array}{l}\text { Número de famílias que participam de } \\
\text { associação/cooperativas }\end{array}$ & 40 & & & 35 & & \\
\hline & & 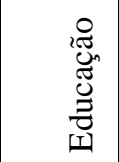 & $\begin{array}{l}\text { Número de entrevistados que possuem } \\
\text { somente o nível fundamental completo }\end{array}$ & 64 & & & 61 & & \\
\hline & & 营 & $\begin{array}{l}\text { Número de famílias que tem acessoa } \\
\text { posto de saúde }\end{array}$ & 100 & & & 100 & & \\
\hline
\end{tabular}

Fonte: Autores.

Sobre os temas Práticas de Manejo do Ambiente Natural e do Usos da Terra, o indicador que surpreendeu positivamente foi o "número de lotes que desenvolvem práticas de conservação ambiental, que apesar de ser considerado intermediário, é um dado importante, pois está no divisor para se tornar um dado negativo, a Lei nº 38 (Mato Grosso, 1995) define o Código Estadual do Meio Ambiente, que tem como meta conscientizar a comunidade da importância do meio ambiente, além disso, a Política Nacional da Reforma Agrária, tem a função de redistribuir as terras, e também, preservar o meio ambiente.

O de maior impacto negativo foi o indicador "Números de lotes que desenvolvem práticas nocivas ao meio ambiente", com valores de 11 e 9, para as mesorregiões Centro-Sul Mato-grossense e Sudoeste Mato-grossense, respectivamente, 
considerado Insustentável, sendo assim, percebe-se que há uma dificuldade em reduzir o uso de produtos que prejudicam o meio ambiente.

Avaliando o cenário, percebe-se que a realidade de Mato Grosso, considerado o estado com maior comercialização de agrotóxicos do Brasil e o maior mercado de defensivos agrícolas, cerca de 27\% do mercado brasileiro (ANA, 2019), nota-se que a utilização desses produtos seja inevitável, já que a fiscalização é ineficiente e o uso aumenta a produtividade da lavoura, além do fato de que produtos orgânicos são mais caros que os produtos cultivados com o uso de agrotóxicos. A questão do uso de agrotóxicos vai além dos problemas gerados pelos consumidores dos produtos, atingindo também a saúde dos agricultores familiares que fazem uso na produção. Monteiro et al (2021), aponta que os "agricultores familiares estão mais vulneráveis aos prejuízos causados por agrotóxicos que outros grupos rurais".

Tratando das dimensões econômicas e sociais, a Escala do Barômetro da Sustentabilidade apontou que o número de agricultores que adquirem renda de atividades fora do lote é potencialmente insustentável (grau 27 para Centro-Sul Matogrossense e 32 para Sudoeste Mato-grossense), esse resultado demonstra que as oportunidades do local não permitem uma abertura para outras fontes de renda. Além do mais, considerando o resultado da Escala do Barômetro da Sustentabilidade do número de agricultores que recebem renda bruta anual total de até $\mathrm{R} \$ 20.000,00$, com grau 56 e 83, percebe-se que os agricultores estão com a produção e fonte de renda limitada a somente a agricultura familiar (PNUD, 2010)

Levando em conta os dados da Escala do Barômetro da sustentabilidade em relação ao número de famílias que recebem algum tipo de Programa social do governo, identifica-se que os indicadores são muito baixo, 9 e 6, respectivamente para as mesorregiões Centro-sul Mato-grossense e Sudoeste Mato-grossense, percebe-se que as famílias não dependem políticas públicas de previdência.

Entretanto, a Política Nacional de Reforma Agrária não está cumprindo seu papel social, de forma racional e adequada (Brasil,1988), pois mesmo com políticas de crédito e assistência técnica, não tem sido capaz de auxiliar o agricultor familiar a retirar a renda da terra em quantidade que atenda às suas necessidades.

O número de entrevistados que possuem o ensino fundamental completo é classificado como potencialmente sustentável para ambas as mesorregiões, sendo 64 para a Centro-Sul Mato-grossense e 61 para Sudoeste Mato-grossense. Esse resultado é muito relevante, pois determina que boa parte da população da região possui acesso ao ensino, e complementando com dados do PNUD (2010), identifica-se que, com o decorrer dos anos, a taxa de analfabetismo tem diminuído e a expectativa de anos de estudo em Mato Grosso, aumentado. Isso caracteriza, que há uma consciência de que a educação é um meio de mudança social. Lima \& Silva (2010) destacam a importância da educação juvenil nos assentamentos em um contexto de educação cidadã, para a vida, valorização da vida no campo para construção de identidades de pertencimento ao meio rural. Soares, Vieira \& Santos (2020) demonstram a importância da educação ambiental para o desenvolvimento sustentável dos assentamentos no âmbito dos Projetos de Desenvolvimento Sustentável (PDS).

Para finalizar os indicadores, a Escala do Barômetro de Sustentabilidade mostra que em relação ao número de famílias que tem acesso ao posto de saúde o grau atingido foi de 100 para as duas mesorregiões, considerado sustentável, esse é um ponto muito importante e demonstra que a Secretaria de Saúde do Estado de Mato Grosso, tem conseguido garantir a universalidade do acesso, visto que segundo a Constituição Brasileira (Brasil, 1988), "a saúde é direito de todos e dever do Estado, garantido mediante políticas sociais e econômicas que visem à redução do risco de doença e de outros agravos, ao acesso universal e igualitário às ações e serviços para sua promoção, proteção e recuperação".

As duas mesorregiões de assentamentos rurais foram representadas em um único gráfico bidimensional (Figura 4), embora com estreita diferença, podem ser consideradas de sustentabilidade intermediária. Assim, nota-se que os projetos de assentamentos rurais da mesorregião Centro-Sul Mato-grossense apresentam ser pouco mais sustentáveis que os projetos de assentamentos rurais da mesorregião Sudoeste Mato-Grossense, devido sua pontuação no grau do barômetro. 
Os resultados obtidos foram: para a mesorregião Centro-Sul Mato-grossense no âmbito do bem-estar do meio ambiente e no bem-estar humano, os graus foram 50 e 47, respectivamente. Em relação a mesorregião Sudoeste Matogrossense os valores obtidos foram: 44 para bem-estar do meio ambiente e 54 para bem-estar humano.

Figura 4 - Barômetro da Sustentabilidade dos Sistemas nas mesorregiões Centro-Sul Mato-grossense e Sudoeste Matogrossense.

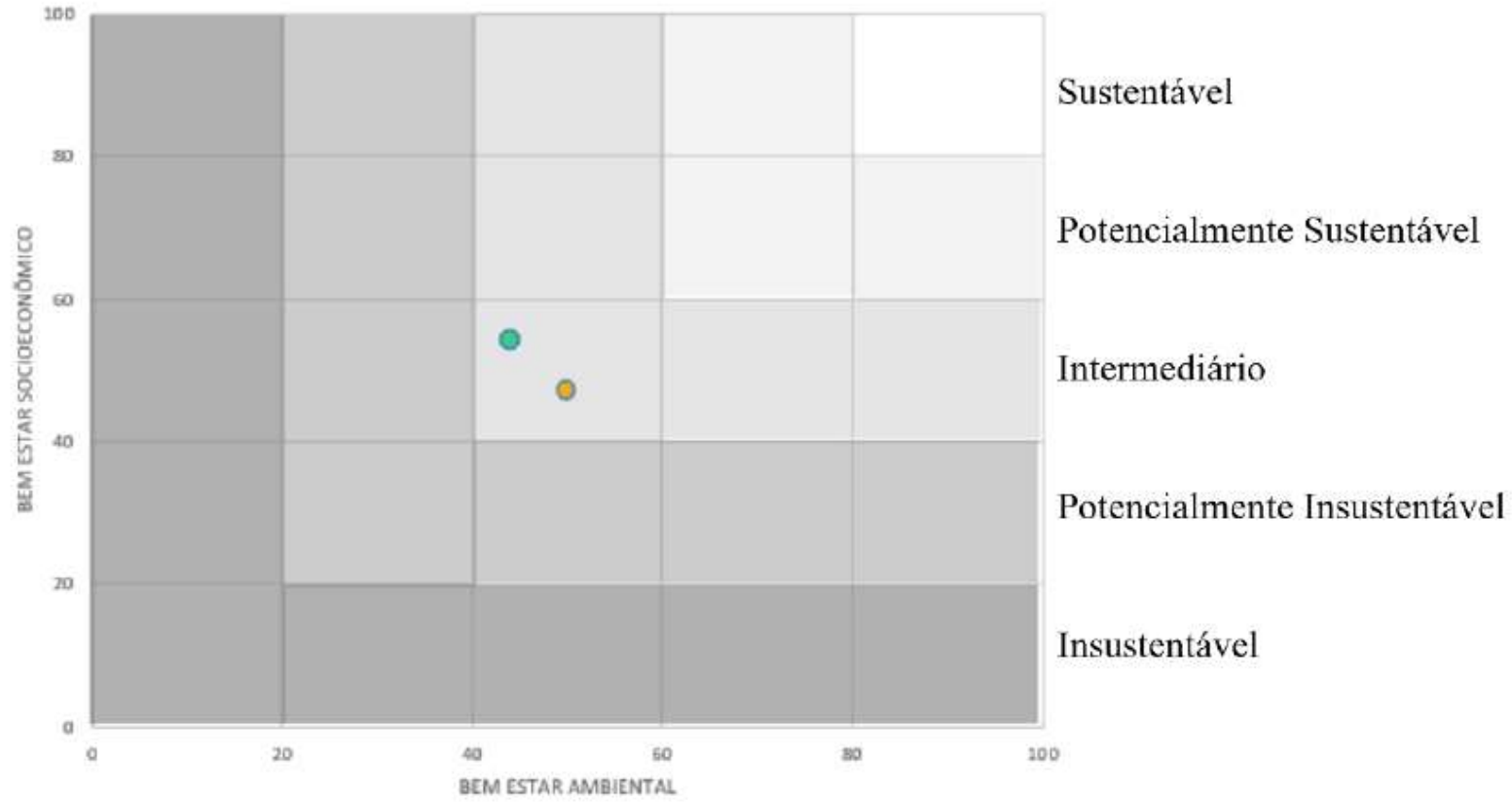

Centro-Sul Mato-Grossense

Sudoeste Mato-Grossense

Fonte: Autores.

A partir desse resultado, nota-se que os fatores que mais influenciaram positivamente para esse resultado foram o alto número de assentamentos que possuem fossa/sumidouro e o acesso a água, que são as condições mínimas definidas pela Organização das Nações Unidas para os direitos humanos. O que influenciou negativamente para o resultado intermediário foram as práticas nocivas os meios ambientes, que apresentou grau insustentável para ambas as mesorregiões e a baixa adesão a medidas sociais do governo.

\section{Considerações Finais}

Cumprindo com o objetivo de analisar os indicadores de sustentabilidade socioeconômica e ambiental dos assentamentos rurais do Estado de Mato Grosso-Brasil, levando em consideração toda a bibliografia estudada e as informações e dados discutidos ao longo do trabalho, é possível concluir que, de maneira geral, os assentamentos rurais do estado de Mato Grosso apresentam o nível de sustentabilidade considerado intermediário, quando analisado o Barômetro da Sustentabilidade no âmbito das mesorregiões Centro-Sul Mato- grossense e Sudoeste Mato-grossense.

Nota-se, a partir dos dados, que os principais resultados positivos e contribuições percebidas são relacionadas, principalmente, aos indicadores de acesso à água e a utilização de fossas, apesar disso, é perceptível a necessidade de avanços no contexto social e econômico dos assentamentos, garantindo melhoria na qualidade de vida, principalmente quando se observa a renda bruta do lote, e a dificuldade de acesso a programas governamentais. 
Por outro lado, o acesso a saúde obteve um elevado grau do indicador, que caracteriza que nesse quesito saúde básica, a população tem sido bem assistida, mas isso não impede a necessidade da implantação de sistema de esgoto, visto que o número de lotes que não possuem rede de esgoto é alto, de forma que com essa implantação, um dos principais objetivos ao se realizar a distribuição de terras seria alcançado, proporcionando uma importante mudança no nível de sustentabilidade do local.

Esses resultados podem ser comparados ao trabalho de Rossetto et al. (2020), em que é possível perceber que apesar do resultado do Barômetro da Sustentabilidade dos assentamentos rurais de Mato Grosso não ser o ideal para o desenvolvimento da sustentabilidade, quando comparado ao estado de Minas Gerais, percebe-se que a realidade mineira apresenta resultado considerado Insustentável, mostrando que se houver um foco no indicadores com maior dificuldade, há grandes chances do Barômetro evoluir facilmente para o parâmetro "Potencialmente Sustentável". Vale destacar, que a pesquisa contribui com a sociedade, com o governo e com as instituições que possuem foco em sustentabilidade rural, pois apresenta dados relevantes para a análise da importância da distribuição de terras.

Por fim, no que se refere as limitações, o estudo limita-se a análise do Barômetro da Sustentabilidade em relação ao estado de Mato Grosso, seria interessante a aplicação desse método em todos os estados brasileiros, para identificar possíveis semelhanças e também as diferenças de cada região acerca dos indicadores locais, além de permitir identificar os recursos que precisam ser investidos em cada estado para melhorar a qualidade do desenvolvimento sustentável dos assentamentos rurais do Brasil

\section{Agradecimentos}

Agradecemos ao Instituto Nacional de Colonização e reforma Agrária (INCRA) e à equipe do Projeto Diagnóstico para Regularização Ambiental dos Assentamentos de Reforma Agrária (RADIS/UFMT).

\section{Referências}

ANA (2021). Agência Nacional de Águas. Conjuntura de Recursos Hídricos - 2019. http://www.snirh.gov.br/.

Brasil. Lei no 11.445, de 05 de janeiro de 2007. (2007). Estabelece diretrizes nacionais para o saneamento básico. Diário Oficial da República Federativa do Brasil.

Brasil. Constituição da República Federativa do Brasil de 1988. Presidência da República. Casa Civil, Brasília, Outubro, 1988.

PNUD. (2010). Programa das Nações Unidas para o Desenvolvimento. Atlas do Desenvolvimento Humano no Brasil. http://atlasbrasil.org.br/2 013/pt/.

Brundtland, G. H. (1987). Nosso Futuro Comum. Relatório Brundtland. Our Common Future:United Nations. Our Common Future: United Nations.

Carvalho, S. P., Ferreira, G. A., Marin, J. O. B., Vargas, F., Belo, A. F. C. F. \& Mendonça, D. C. (2009). Reforma agrária: a realidade de um assentamento rural. CampoTerritório: Revista de Geografia Agrária, Uberlândia, 4(8), 67-97.

Gallopin, G. C. (1996). Environmental and sustainability indicators and the concept of situational indicators. A system approach. Environmental Modelling \& Assessment, 1, 101-117.

Guerra, R. M. N. (2002). É possível atingir a sustenatbilidade nos assentaemntos de Reforma Agrária na Amazônia Legal? O caso do PDS São Salvador no Estado do Acre. (Dissertação). Universidade de Brasília, Brasília, DF.

IBGE. (2013). Instituto Brasileiro de Geografia e Estatística. Estatística. Características étnico-raciais da população: classificações e identidades.

IBGE. (2016). Instituto Brasileiro de Geografia e Estatística. Estatística. Síntese de indicadores sociais: uma análise das condições de vida da população brasileira. https://biblioteca.ibge.gov.br/visualizacao/livros/liv98965.pdf.

IBGE. (2017). Instituto Brasileiro de Geografia e Estatística.. Censo Agropecuário 2017. Resultados Preliminares.

Kronemberger, D. M. P., Carvalho, C. D., \& Junior, J. C. (2012). Indicadores de sustentabilidade em pequenas bacias hidrográficas: uma aplicação do "Barômetro da Sustentabilidade" à Bacia do Jurumirim (Angra dos Reis/RJ). Geochimica Brasiliensis, 18(2), 86-98.

Mato Grosso (1995). Lei Complementar n 38, de 21 de novembro de 1995. Dispõe sobre o Código Estadual do Meio Ambiente e dá outras providências. Diário Oficial do Estado de Mato Grosso. 
Research, Society and Development, v. 10, n. 15, e532101523320, 2021

(CC BY 4.0) | ISSN 2525-3409 | DOI: http://dx.doi.org/10.33448/rsd-v10i15.23320

Mato Grosso. (2020). Lei Estadual $\mathrm{n}^{\circ}$ 11.088, de 09 de março de 2020. Dispõe sobre a Política Estadual de Recursos Hídricos, institui o Sistema Estadual de Recursos Hídricos e dá outras providências. Diário Oficial do Estado de Mato Grosso.

Monteiro, P. H. S., Gonçalves, L. M., Santos, L. S., Fraga, L. K., Outeiro, M. T., Zboralski, A. R. \& Godoy, W. I. 2021. Os perigos dos agrotóxicos para famílias agricultoras - Uma revisão integrativa. Research, Society and Development, 10(9).

Lima, G. C. \& Silva, S. B. (2010). Juventudes rurais e processos educativos. Research, Society and Development, 9(1), e98591110691.

ONU. Organização das Nações Unidas. Resolução A/RES/64/292. The human right to water and sanitation.

Peroni, J. B., Carvalho, L. H. \& Lannes, L. S. (2021). Aspectos de qualidade da água e saneamento básico em um assentamento rural no interior de São Paulo: diagnóstico e perspectivas para a melhoria da qualidade sócio-ambiental. Research, Society and Development, 10(2), e58510918414.

PNUD. (2010). Programa das Nações Unidas para o Desenvolvimento. Atlas do Desenvolvimento Humano no Brasil. http://www.atlasbrasil.org.br/

Prescott-Allen, R. (2001). The wellbeing of nations. Island Press.

Prescott-Allen, R. (1997). Barometer of Sustainability: Measuring and communicating wellbeing and sustainable development. IUCN, Gland, CH.

Rossetto, O. C., Durante, L. C., Venere, P. C., Rabelo, O. da S.,.Teixeira, R. F. da S., Dalla Nora, G., Lima, D. M. D. F. de, Lima Neto, J. T. de \& Santos, C. A S. Alves. (2015). Indicators of socio-economic and environmental sustainability of agrarian reform settlements in Minas Gerais - Brazil. International Journal of Development Research. 10(10), 41273-41285.

Sá-Silva, J.R., Almeida, C. D. \& Guindani, J. F. (2009). Pesquisa documental: pistas teóricas e metodológicas. Revista Brasileira de História \& Ciências Sociais, 1(1), 1- 15 .

Silva, M. A. D. (2001). Assentamento e sustentabilidade. Sociedade e Cultura, 4(1), 85-103.

Silva, V. C. S., Vieira, I. C. G. (2016). Barômetro da Sustentabilidade aplicado a assentamentos rurais do leste do Estado do Pará, Brasil. Desenvolvimento e Meio Ambiente, 36, 201-221.

Soares, F. I. L., Vieira, T. A. \& Santos, M. M. C. (2020). Educação Ambiental como aliada à sustentabilidade em Projetos de Desenvolvimento Sustentável (PDS). Research, Society and Development, 9(12), e45891211222.

Van Bellen, H. M. (2007). Indicadores de Sustentabilidade: uma análise comparativa. (2a ed.), FGV Editora. 